\section{Beeinflusst der TNF-Haplotyp den Akne-Schweregrad?}

\author{
In der Vergangenheit konnten bereits verschiedene Single \\ Nucleotide Polymorphisms (SNPs) identifiziert werden, die mit \\ der Entstehung der Acne vulgaris in Zusammenhang stehen. \\ Welche Effekte die SNPs des TNF-Gens auf die Ausprägung \\ und den Verlauf der Erkrankung haben, ist jedoch unklar. \\ Dermatology 2014; 228: 152-157
}

Das TNF-Gen kodiert für den TumorNekrose-Faktor- $\alpha$ (TNF- $\alpha)$, ein Zytokin, das an Entzündungsprozessen beteiligt ist. Die Fall-Kontroll-Studie der Forscher um I. Grech fand zwischen 2009 und 2011 statt und schloss 185 Patienten mit Acne vulgaris und 165 gesunde Kontrollen ein. Aus dem Blut der Probanden wurde genomische DNA isoliert, auf deren Basis eine Analyse ausgewählter SNPs erfolgte (Positionen $-376,-308$ und -238 innerhalb der Promotorregion des TNF-Gens).

\section{GAG-Haplotyp mit Erkrankungsrisiko assoziiert $\nabla$}

Der Anteil weiblicher Studienteilnehmer betrug in der Patienten- und Kontrollgruppe jeweils 84,9 bzw. 84,6\%. Hinsichtlich des Genotyps GA/AA an den Positionen -376 und -238 innerhalb des TNFPromotors zeigten Patienten und Kontrollen keine Unterschiede. Allerdings war die Häufigkeit des GA/AA-Genotyps an der Position -308 bei den Patienten höher. Die
Autoren ermittelten innerhalb der Patientengruppe eine höhere Frequenz des GAG-Haplotyps als in der Kontrollgruppe (16,8 vs. 9,7\%), das Signifikanzniveau lag allerdings im Grenzbereich ( $p=0,059)$. Bei Männern war die Existenz des GGG-Haplotyps mit einem früheren Einsetzen der Akne assoziiert. Die Autoren vermuteten, dass eventuell eine Assoziation zwischen dem polyzystischen Ovarialsyndrom, bestimmten TNF-Haplotypen und einer Acne conglobata besteht. Die vorliegenden Studiendaten konnten die Hypothese allerdings nicht bestätigen.

\section{Fazit}

Die Studienergebnisse unterstreichen den Zusammenhang zwischen Polymorphismen im TNF-Gen und der Acne vulgaris. Träger des GAG-Haplotyps weisen bspw. ein erhöhtes Erkrankungsrisiko auf. Der GGG-Haplotyp wiederum geht bei Männern mit einem früheren Krankheitsbeginn einher. Relativiert werden die Ergebnisse nach Meinung der Autoren durch den geringen Anteil an männlichen Studienteilnehmern.

Dr. Frank Lichert, Weilburg

\title{
Staphylococcus aureus schwächt die Immunantwort
}

Eine Infektion mit S. aureus stört bei Neurodermitis die myeloisch-monozytären Zellen (Myeloid-Derived Suppressor Cells, MDSCs): Eine hohe Anzahl an S. aureus führt über einen Signalweg zur Bildung großer Mengen an MDSCs, wodurch hilfreiche Immunantworten unterdrückt werden. Das Team von Prof. T. Biedermann von der Klinik für Allergologie und Dermatologie am Klinikum rechts der Isar und der Universität Tübingen konnte im Tiermodell zeigen, wie Infektionen durch S. aureus den Krankheitsverlauf der Neurodermitis zusätzlich verschlechtern. Die Forschungsergebnisse wurden im November in der Fachzeitschrift Immunity veröffentlicht.

\section{Abwehrstrategie lahmgelegt $\nabla$}

Als Barriere für krankmachende Keime ist die Haut mit sog. Toll-like-Rezeptoren ausgestattet. Diese Rezeptoren erkennen
Substanzen von Bakterien wie z. B. Oberflächenproteine und aktivieren daraufhin das Immunsystem. In ihren Experimenten beobachteten die Wissenschaftler, dass Zellwandproteine von S. aureus auf der Haut die Bildung von MDSCs über einen neuen Signalweg auslösten. Unter normalen Bedingungen regulieren MDSCs Immunantworten und Entzündungen, indem sie diese im richtigen Moment unterdrücken bzw. beenden. In den Experimenten führten die vielen Bakterienproteine von $\mathrm{S}$. aureus auf der Haut aber zu einer übermäßigen Bildung von MDSCs - die Folge: auch hilfreiche Immunantworten in der Haut wurden unterdrückt. „Für den Kampf gegen die Erreger ist das eine sehr ungünstige Entwicklung. Die MDSCs unterdrücken schützende Abwehrstrategien in der Haut und verstärken so die Folgen der S. aureus-Infektion“, so Biedermann.
Neben dem Tiermodell untersuchten die Forscher auch 33 Patienten mit Neurodermitis und stellten fest, dass sie im Vergleich zu Gesunden erhöhte Mengen an MDSCs im Blut und in der Haut hatten. „Diese klinische Beobachtung bestätigt unsere Ergebnisse. Die MDSCs scheinen gerade bei Neurodermitis-Patienten in großen Mengen vorhanden zu sein und so die Immunantworten der Haut zu unterdrücken - mit negativen Folgen für den Krankheitsverlauf.“, erklärt Biedermann.

„Gerade schwer betroffene Patienten leiden sehr unter der chronischen Entzündung, dem Juckreiz und der Stigmatisierung durch die Umwelt. Eine frühzeitige und konsequente Behandlung der Patienten, die auch die Bakterien miteinschließt, ist sehr wichtig“, betont er. Mit seinem Team will er in nächsten Schritten untersuchen, wie Entzündungen durch MDSCs normalerweise beendet werden und dieses Wissen für neue Therapieansätze gegen entzündliche Hautkrankheiten wie Neurodermitis nutzen.

Nach einer Mitteilung

der Technischen Universität München 